\title{
The use of ultrasound in establishing COVID-19 infection as part of a trauma evaluation
}

\author{
Jose Diaz-Miron ${ }^{1}$ (D) Marina L. Reppucci ${ }^{1}$. Jason Weinman ${ }^{2} \cdot$ Alexander Kaizer $^{3}$ - Aparna Annam² \\ Jonathan Orsborn ${ }^{4} \cdot$ Lauren Steward $^{5} \cdot$ Juliana Wilson $^{6} \cdot$ Denis Bensard $^{1}$
}

Received: 14 October 2021 / Accepted: 29 November 2021 / Published online: 6 January 2022

(c) American Society of Emergency Radiology 2021

\begin{abstract}
Purpose The use of lung ultrasound for diagnosis of COVID-19 has emerged during the pandemic as a beneficial diagnostic modality due to its rapid availability, bedside use, and lack of radiation. This study aimed to determine if routine ultrasound (US) imaging of the lungs of trauma patients with COVID-19 infections who undergo extended focused assessment with sonography for trauma (EFAST) correlates with computed tomography (CT) imaging and X-ray findings, as previously reported in other populations.

Methods This was a prospective, observational feasibility study performed at two level 1 trauma centers. US, CT, and X-ray imaging were retrospectively reviewed by a surgical trainee and a board-certified radiologist to determine any correlation of imaging findings in patients with active COVID-19 infection.

Results There were 53 patients with lung US images from EFAST available for evaluation and COVID-19 testing. The overall COVID-19 positivity rate was 7.5\%. COVID-19 infection was accurately identified by one patient on US by the trainee, but there was a $15.1 \%$ false-positive rate for infection based on the radiologist examination.

Conclusions Evaluation of the lung during EFAST cannot be used in the trauma setting to identify patients with active COVID-19 infection or to stratify patients as high or low risk of infection. This is likely due to differences in lung imaging technique and the presence of concomitant thoracic injury.
\end{abstract}

Keywords Trauma · COVID-19 · Extended focused assessment with sonography in trauma $\cdot$ Lung ultrasound · Thoracic trauma

\section{Introduction}

The COVID-19 pandemic resulted in a unique situation and challenge for frontline healthcare providers who strive to provide optimal, prompt medical care while minimizing personal exposure in acute scenarios. As the burden of disease and risk of exposure increased, clinicians incorporated other modalities to bypass the limitations of the most commonly used diagnostic tools, computed tomography (CT), chest X-ray, and polymerase chain reaction (PCR) tests, to expeditiously determine those with high probability of

Jose Diaz-Miron and Marina L. Reppucci contributed equally to this work.

Jose Diaz-Miron

jose.diaz-miron@childrenscolorado.org

Extended author information available on the last page of the article active infection. CT is the imaging gold standard to assess the severity of pulmonary manifestations of the COVID-19 infection, and the imaging patterns have been well characterized in the literature, with multiple studies showing high rates of sensitivity $[1,2]$. As the pandemic worsened, the use of lung ultrasound (US) emerged as a beneficial diagnostic modality due to its rapid availability, bedside use, and lack of radiation $[3,4]$. Multiple studies have reported lung US to have a very high sensitivity $(89-100 \%)$ with a reliability comparable to that of CT [5-7]. Interest in its use is further underscored by the fact that it is inexpensive and easy to disinfect, a priority for low-resource settings, which are currently the ones most affected by the pandemic.

One patient population that benefits from real-time US evaluation is trauma patients. Advanced trauma life support (ATLS) algorithms place the utmost importance on rapid and systematic evaluation of the injured patient. During this initial evaluation, patients may also receive adjunct imaging 
modalities like the extended focused assessment with sonography for trauma (EFAST) exam designed to discover lifethreatening findings that require urgent interventions. During this exam, the pericardial, perisplenic, perihepatic, and pelvic areas are assessed for free fluid, and the anterior and lateral pleural spaces are assessed for pneumothorax or hemothorax. Given that this is routine in ATLS algorithm, expansion of the utility of lung US for detection of active infection or to stratify trauma patients into high or low risk for active infection could prove useful.

The aim of this study was to determine the feasibility of using lung ultrasound for the diagnosis of pulmonary manifestations of COVID-19 in the context of trauma patients undergoing routine EFAST imaging in the emergency department (ED). We sought to determine if US imaging of the lungs of trauma patients with COVID-19 infections correlates with $\mathrm{CT}$ imaging and $\mathrm{X}$-ray findings, as previously reported in other populations.

\section{Methods}

This was a prospective, observational feasibility study with a retrospective component performed at Children's Hospital Colorado (CHCO) and the University of Colorado Hospital (UCH) at the Anschutz Medical Center. Data was collected on patients between August 2020 and March 2021. CHCO is an American College of Surgeons (ACS) and state-verified level 1 pediatric trauma center (PTC). The UCH is an ACS and state-verified level 1 adult trauma center. Both institutions serve a large metropolitan area. In addition, $\mathrm{CHCO}$ is the state's only level 1 PTC and serves seven adjacent states. The state of Colorado has experienced more than 500,000 confirmed cases of COVID-19 since the onset of the pandemic with over 30,000 resulting in hospitalization. The state experienced its most significant "surge" during the months of November-January, with 7-day averages nearing 5000 cases [8]. The Colorado Multiple Institutional Review Board (COMIRB) approved this study.

All patients who met trauma criteria activation or alert at their respective institutions and underwent EFAST as part of their standard trauma workup were included in analysis. EFAST evaluations were performed by members of the trauma team, most commonly emergency medicine or general surgery residents, in the ED for the purposes of trauma evaluation, and practitioners were blinded to COVID-19 status of the patient at time of evaluation. They were performed in the standardized trauma manner, with imaging limited to the anterior and lateral lung fields due to the immobilization of trauma patients [9]. As EFAST is a standard part of trauma evaluation, waiver of consent was approved by the COMIRB. Patient lists of trauma evaluations from each institution were provided by each respective registrar and reviewed by the study staff to identify patients who met inclusion criteria. Patients were excluded if they did not have a COVID-19 test or if their sonographic examination was limited to a FAST evaluation, without evaluation of lung windows.

Patient variables retrospectively collected included location of trauma evaluation, radiographic findings, sonographic findings, and COVID-19 status by a single abstractor. Radiographic data collected included findings on chest $\mathrm{X}$-ray or chest CT. Lung images from EFAST examinations were independently reviewed by a surgery resident with 3 years of clinical experience to simulate the real-life trauma surgical consultant typical ultrasonographic experience and by a board-certified radiologist. Only available in the picture archiving and communication system (PACS) and only cine images were reviewed. Images were assessed for the presence of diffuse pleural line abnormalities, subpleural consolidations, white lung areas or thick, or irregular vertical artifacts. Based on these findings, the image reviewer independently categorized the patient as having a "low probability" or "high probability" of active infection based on presence of less than two findings or two or more findings, respectively. Imaging was interpreted without knowing the patient's COVID-19 status. COVID-19 results were subsequently recorded based on PCR tests performed at time of patient's trauma evaluation.

All data was summarized as count (percent) given all variables are dichotomous in nature. For CT and X-ray results, the denominator used to calculate percentages is restricted to those with the given imaging. Comparisons between the findings and COVID-19 test results are conducted with Fisher's exact test to account for small counts. Significance was set at $p<0.05$. Data analyses were conducted using $\mathrm{R}$ version 3.4.1 software (R Foundation for Statistical Computing, Vienna, Austria, http://www.R-project.org/).

\section{Results}

During the study period, there were 317 patients who were evaluated with an EFAST and had a COVID-19 test performed, with only 17 of these having a positive COVID-19 test $(5.05 \%)$. Upon review of the 317 who received EFAST evaluation and had a COVID-19 imaging, 71 (22.3\%) patients had images available in PACS. The remaining 246 patients did not have images saved to PACS for retrospective review by the research team, despite documentation of an EFAST being completed. Among the 71 patients with images available, 18 patients had saved images of quality too poor to review and were omitted from the study. This left 53 patients for inclusion in study analysis.

Among those included in analysis, 45 patients were evaluated at the $\mathrm{UCH}$ and eight patients were evaluated at $\mathrm{CHCO}$. 
On trauma evaluation, $37(69.8 \%)$ patients had a chest CT completed and $49(92.5 \%)$ had a chest X-ray completed. Of these patients, $7.5 \%(n=4)$ had a positive COVID-19 test.

On chest X-ray, there was no differences in findings between patients with negative or positive COVID-19 test results. Rib fractures were found in three (6.1\%) patients, including one who was COVID-19 positive. Unilateral ground-glass opacities were visualized in four $(8.2 \%)$ patients, including one patient with COVID-19 (Table 1).

On chest CT, there was no statistically significant differences in findings between COVID-19-positive and COVID19-negative patients. There were eight (15.1\%) patients with rib fractures on CT. Two COVID-19-positive patients were found to have rib fractures, one with unilateral rib fractures and one with bilateral rib fractures. Full CT scan findings are displayed in Table 2.

EFAST images were independently reviewed by a surgical trainee and attending radiologist. Diffuse pleural line abnormalities were the most common finding identified by the trainee on five (9.4\%) of the EFAST images. This finding was more common in COVID-19-positive patients ( $50.0 \%$ vs $6.1 \%, p=0.04)$. The trainee categorized two (3.8\%) patients as having high probability of infection based on EFAST findings. One of these patients was COVID-19 positive. Full EFAST findings are available in Table 3.

Diffuse pleural line abnormalities were the most common finding in EFAST images evaluated by the attending radiologist and were identified in eight $(15.1 \%)$ patients. All of these patients were COVID-19 negative. Overall, the attending radiologist categorized eight patients as having high probability of infection, all of whom were ultimately COVID-19 negative, resulting in a $15.1 \%$ false-positive rate (Table 4). There were no statistically significant differences in EFAST findings between COVID-19-positive and COVID-19-negative patients. Representative images of lung US and CT is provided in Figs. 1 and 2, highlighting imaging findings from traumatic injury that
Table 1 Chest X-ray findings, stratified by COVID-19 status

Table 2 Chest CT findings, stratified by COVID-19 status

Table 3 EFAST trainee findings, stratified by COVID19 status

\begin{tabular}{lllll}
\hline & $\begin{array}{l}\text { COVID-19 posi- } \\
\text { tive }(n=4)\end{array}$ & $\begin{array}{l}\text { COVID-19 nega- } \\
\text { tive }(n=45)\end{array}$ & $\begin{array}{l}\text { Total population } \\
(n=49)\end{array}$ & $p$-value \\
\hline Unilateral consolidation & $0(0.0 \%)$ & $2(4.4 \%)$ & $2(4.1 \%)$ & 1.000 \\
Bilateral consolidations & $0(0.0 \%)$ & $1(2.2 \%)$ & $1(2.0 \%)$ & 1.000 \\
Unilateral ground-glass opacities & $1(25.0 \%)$ & $3(6.7 \%)$ & $4(8.2 \%)$ & 0.297 \\
Bilateral ground-glass opacities & $0(0.0 \%)$ & $2(4.4 \%)$ & $2(4.1 \%)$ & 1.000 \\
Unilateral rib fractures & $1(25.0 \%)$ & $2(4.4 \%)$ & $3(6.1 \%)$ & 0.230 \\
Bilateral rib fractures & $0(0.0 \%)$ & $0(0.0 \%)$ & $0(0.0 \%)$ & 1.000 \\
\hline
\end{tabular}

\begin{tabular}{llllc}
\hline & $\begin{array}{l}\text { COVID-19 } \\
\text { positive }(n=4)\end{array}$ & $\begin{array}{l}\text { COVID-19 } \\
\text { negative }(n=33)\end{array}$ & $\begin{array}{l}\text { Total popula- } \\
\text { tion }(n=37)\end{array}$ & $p$-value \\
\hline Unilateral ground-glass opacities & $1(25.0 \%)$ & $4(12.1 \%)$ & $5(13.5 \%)$ & 0.456 \\
Bilateral ground-glass opacities & $0(0.0 \%)$ & $4(12.1 \%)$ & $4(10.8 \%)$ & 1.000 \\
Ground-glass opacities in central location & $0(0.0 \%)$ & $1(3.0 \%)$ & $1(2.7 \%)$ & 1.000 \\
Ground-glass opacities in the periphery & $1(25.0 \%)$ & $3(9.1 \%)$ & $4(10.8 \%)$ & 0.380 \\
Unilateral lung consolidation & $0(0.0 \%)$ & $3(9.1 \%)$ & $3(8.1 \%)$ & 1.000 \\
Lung consolidations in the periphery & $0(0.0 \%)$ & $1(3.0 \%)$ & $1(2.7 \%)$ & 1.000 \\
Unilateral rib fractures & $1(25.0 \%)$ & $5(15.2 \%)$ & $6(16.2 \%)$ & 0.524 \\
Bilateral rib fractures & $1(25.0 \%)$ & $1(3.0 \%)$ & $2(5.4 \%)$ & 0.207 \\
\hline
\end{tabular}

\begin{tabular}{lllll}
\hline & $\begin{array}{l}\text { COVID-19 posi- } \\
\text { tive }(n=4)\end{array}$ & $\begin{array}{l}\text { COVID-19 nega- } \\
\text { tive }(n=49)\end{array}$ & $\begin{array}{l}\text { Total population } \\
(n=53)\end{array}$ & $p$-value \\
\hline Diffuse pleural line abnormalities & $2(50.0 \%)$ & $3(6.1 \%)$ & $5(9.4 \%)$ & 0.040 \\
Subpleural consolidations & $0(0.0 \%)$ & $0(0.0 \%)$ & $0(0.0 \%)$ & 1.000 \\
White lung areas & $1(25.0 \%)$ & $1(2.0 \%)$ & $2(3.8 \%)$ & 0.147 \\
Thick, irregular vertical artifacts & $0(0.0 \%)$ & $1(2.0 \%)$ & $1(1.9 \%)$ & 1.000 \\
High probability active infection & $1(25.0 \%)$ & $1(2.0 \%)$ & $2(3.8 \%)$ & \\
\hline
\end{tabular}


Table 4 EFAST attending findings, stratified by COVID19 status

\begin{tabular}{lllll}
\hline & $\begin{array}{l}\text { COVID-19 posi- } \\
\text { tive }(n=4)\end{array}$ & $\begin{array}{l}\text { COVID-19 nega- } \\
\text { tive }(n=49)\end{array}$ & $\begin{array}{l}\text { Total population } \\
(n=53)\end{array}$ & $p$-value \\
\hline Diffuse pleural line abnormalities & $0(0.0 \%)$ & $8(16.3 \%)$ & $8(15.1 \%)$ & 1.000 \\
Subpleural consolidations & $0(0.0 \%)$ & $4(8.2 \%)$ & $4(7.5 \%)$ & 1.000 \\
White lung areas & $0(0.0 \%)$ & $4(8.2 \%)$ & $4(7.5 \%)$ & 1.000 \\
Thick, irregular vertical artifacts & $0(0.0 \%)$ & $3(6.1 \%)$ & $3(5.7 \%)$ & 1.000 \\
High probability active infection & $0(0.0 \%)$ & $8(16.3 \%)$ & $8(15.1 \%)$ & \\
\hline
\end{tabular}

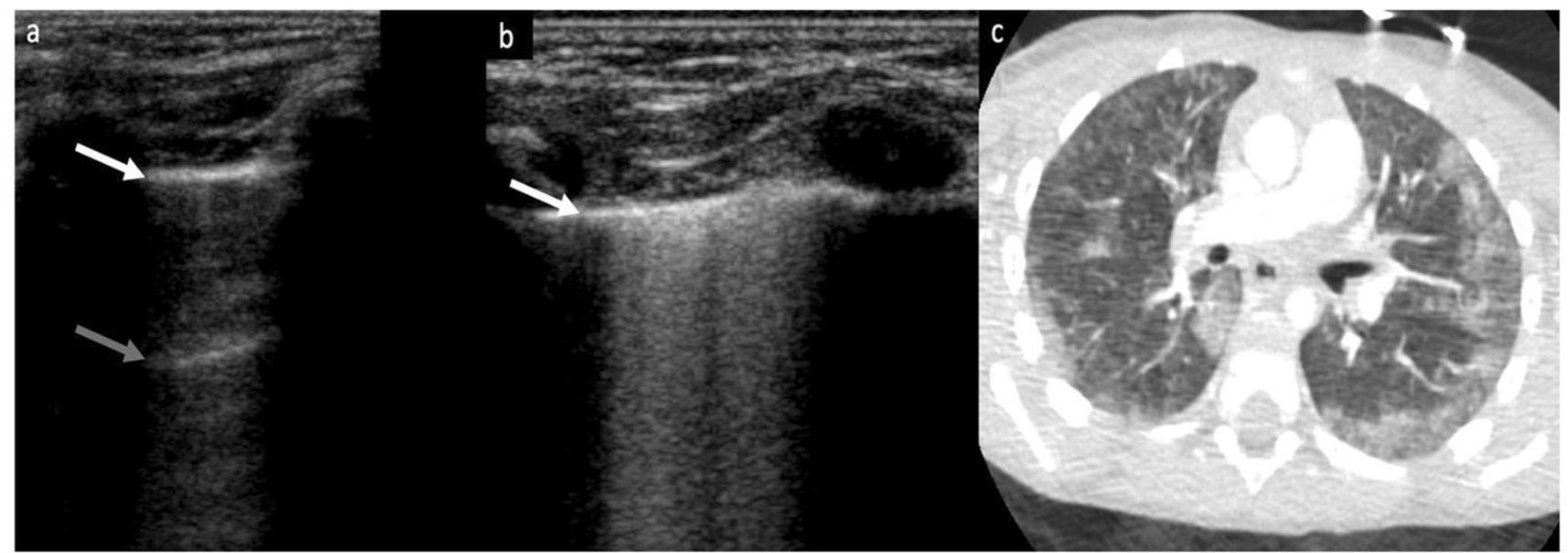

Fig. 1 A 4-year-old male in rollover motor vehicle accident with negative COVID-19 result. a EFAST images of the right lung demonstrate normal lung parenchyma with pleural line (white arrow) and preserved A-lines (gray arrow). b EFAST images of the left lung demonstrate a white lung appearance with increased echogenicity deep to the pleural line (white arrow) and loss of the A-lines seen in the right lung. c Motion degraded axial CT image of the lung in the same patient demonstrate peripheral areas of ground-glass opacity and consolidation greater on the left than the right
Fig. 2 A 69-year-old male in head-on motor vehicle collision. a EFAST images of the left lung demonstrate a loss of A-lines with mildly increased echogenicity deep to the pleural line (white arrow). b Axial CT image of the lung in the same patient demonstrate peripheral areas of consolidation bilaterally

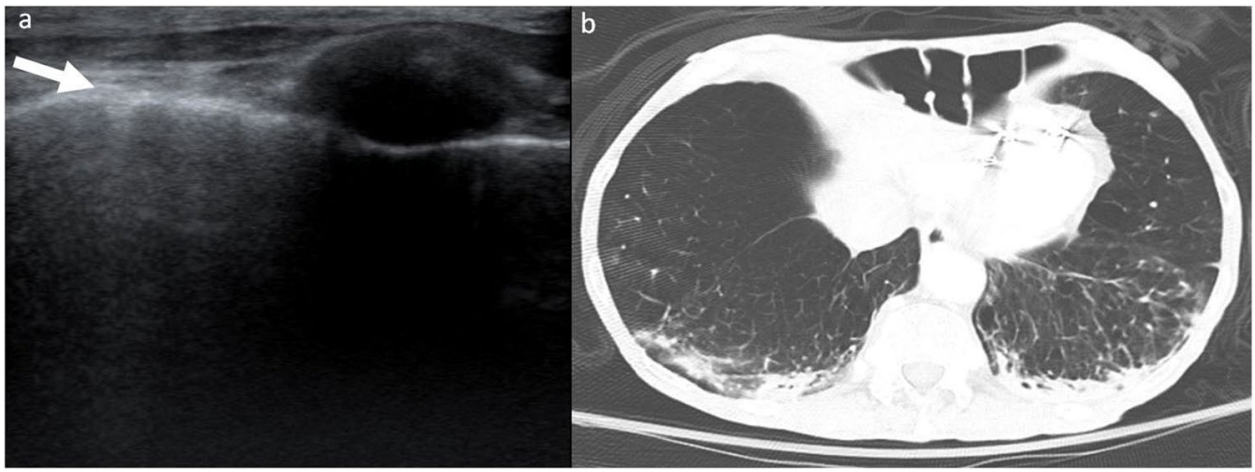

mimic characteristic of active infection in pediatric and adult patients without COVID-19.

The four patients who were found to be COVID-19 positive all had associated thoracic injuries. The first patient presented after a fall and was found to have unilateral rib fractures, with ground-glass opacities on CT scan. The second patient also presented after a fall and was found to have bilateral rib fractures and unilateral clavicle rib fracture. CT scan was remarkable for unilateral ground-glass and consolidative opacities. The remaining two COVID19-positive patients presented after assault. The first patient was found to have unilateral rib fractures with adjacent atelectasis; both CT and X-ray were otherwise unremarkable. The final patient sustained multiple stab wounds resulting in an upper lobe lung laceration, unilateral rib fractures with hemothorax, and pneumothorax visualized on $\mathrm{CT}$ and $\mathrm{X}$-ray. 


\section{Discussion}

This study suggests that lung US imaging on routine EFAST cannot be used to identify patients with active COVID-19 infection or to stratify patients as high or low risk of infection. Our findings that lung US from an EFAST exam is not a reliable screening tool for COVID19 infection are in opposition to multiple prior studies that have shown dedicated lung point of care ultrasound (POCUS) to be a reliable and sensitive tool for identifying patients with COVID-19. Our study was limited to patients who presented with traumatic injury and found US to be a poor tool for identifying active infection. In fact, EFAST imaging was identified as presenting high probability of infection in only one COVID-19-positive patient by the surgical trainee. The attending radiologist identified eight patients as having high probability of active infection, but all were ultimately COVID-19 negative. This resulted in identifying $15.1 \%$ of patients as falsely positive. Prior studies have shown US to be a highly sensitive test, with values exceeding $80 \%$ [5-7, 9]. However, EFAST imaging in our analysis more frequently identified COVID-19-negative patients as having a high probability of infection.

Prior studies assessing the diagnostic ability of POCUS in the setting of active COVID infection have found promising results. The first US evaluation of COVID was a case report from Italy, which identified findings of bilateral, diffuse pleural line abnormalities, subpleural consolidations, white lung areas, and thick, irregular vertical artifacts [10]. The diagnostic accuracy of POCUS was evaluated in a prospective study from the Netherlands, which found it to have sensitivity of $100 \%$ and a specificity of $76 \%$ in patients without cardiac disease and sensitivity of $89 \%$ and specificity of 59\% in those with [11]. Its diagnostic accuracy has been validated by further studies, which have shown US findings correlate well with CT scan findings [5-7]. It should be noted that all these studies were performed in patients with either known COVID-19 infection or suspected COVID-19 infection based on presenting symptoms, which differs from our patient population who underwent lung sonography for the purpose of trauma evaluation. It should be emphasized that though basic clinical history is part of the secondary survey of ATLS, questions related to presenting COVID-19 symptoms, such as cough and fever, are not routinely asked in the setting of trauma, with the exception of dyspnea in the setting of thoracic trauma.

Our study is unique in that it was limited to trauma patients who presented to the ED for reasons other than respiratory symptoms or for suspected COVID-19 infection. Our study did not account for injury severity, but it should be noted that the pandemic resulted in a reluctance of trauma patients to present to the ED, especially during periods of high COVID-19 rates, with rates decreasing by approximately $50 \%$ compared to years prior to the pandemic $[12,13]$. For example, largely due to stay at home orders and/or compliance to social distancing, there were reported declines in traffic and traffic-related injuries [14]. Though decreased rates of all trauma patients were reported, there have been mixed reports of increases in severity of presenting trauma, with some centers noting increases in injury severity and some reporting no change $[13,15]$. Our study did not account for trauma trends, but it is important to recognize the impact the pandemic has had on trauma presentations.

The significance of trauma trends is related to its impact on EFAST imaging. Within the context of trauma, POCUS has been shown to have good sensitivity for traumatic pneumothorax and hemothorax, the two injuries of interest during EFAST assessment in the emergency setting [16]. This diagnosis is made by the absence of lung-sliding. However, findings consistent with COVID-19 infection have never been studied in the context of concomitant injuries, and it is unknown how the presence of thoracic injuries, such as rib fractures, pulmonary contusions, pneumothorax, or hemothorax, may affect the expected US findings in active infection. It is known, however, that pleural line may be lost in hemothorax, pneumothorax, and contusions, which would limit the diagnostic ability of US to detect lung pathology in the presence of such injuries. Our results suggest that when thoracic injuries are present, the accuracy of POCUS for the diagnosis of COVID-19 is poor, possibly due to the changes secondary to traumatic injuries. It should be noted that there have been several reports of COVID-19 infection mimicking pulmonary contusion on CT imaging, specifically due to similarities in lung parenchymal changes such as peripheral subpleural consolidation and ground-glass opacity [17-19]. The similarities have not been studied in explicitly in US, though it is known both pathologies can exhibit B-lines [20]. This may explain the reason for a $15.1 \%$ false-positive rate based on the radiologist's examination. Furthermore, prior studies have supported that cardiopulmonary comorbidities decreases the sensitivity and specificity of POCUS in identifying COVID-19 [11]. This suggests that lung US may only have high specificity in patients without any other lung pathologies.

Prior studies that confirm the diagnostic accuracy of POCUS for identifying COVID-19 infection utilized strict imaging protocols that involved evaluation of multiple zones of the lung, including posterior, anterior, and lateral regions $[5,11,21]$. This protocol has been proven to detect lung parenchymal changes. Importance is placed on imaging the posterior lung fields because ground-glass opacities and consolidations associated with COVID-19 are known to have a tendency for peripheral and posterior lung distributions 
[22-24]. However, the protocol for EFAST imaging of the lungs differs. EFAST assessments serve to identify lifethreatening injuries in trauma patients and do so with substantial accuracy [25]. Since its purpose is to identify hemothorax and pneumothorax, its evaluation is limited to the anterior and lateral chest [9]. Some studies even suggest that single views of the anterior thorax are sufficient to diagnose pneumothorax [26]. The primary purpose of EFAST is to determine if life-threatening injuries exist in an expeditious manner. Due to the acuity of trauma patients' presentation and their associated immobility, posterior lung windows are not assessed. However, in the context of COVID-19 infection, Rizzetto et al. evaluated the sensitivity of different lung fields and found the sensitivity of anterior lung fields to be significantly less than that of posterior lung fields [5]. This likely partially explains the poor diagnostic ability of EFAST examinations in this study. As the anatomical location of the thoracic pathology of interest differs from the pattern exhibited in COVID-19 infections, the ability of EFAST to detect the virus is limited.

This study is not without limitations. There were a significant number of patients who had EFAST examinations performed, but their images were not available on PACS. Furthermore, not all patients received COVID-19 testing on admission despite EFAST imaging and were excluded from analysis. This limited our sample size for analysis and may have skewed our results which ultimately only included four COVID-19-positive patients. This small number of cases results in low statistical power to detect potentially significant differences. Furthermore, US is operator dependent. Our study focused on imaging performed as EFAST during the trauma setting. These images are usually performed by surgical or emergency medicine trainees, for the purpose of identifying hemothorax or pneumothorax. This may have limited the quality of US imaging for interpretation. However, because the purpose of this study was a feasibility study, we did not attempt to alter normal processes during trauma activations to result in higher quality imaging. Processes may be different at other institutions and our findings may not be generalizable to all institutions. Finally, it is suspected that pulmonary contusions, a common thoracic injury, can mimic COVID-19 infection on ultrasound, which would impair its ability to identify infection in the setting of trauma patients.

\section{Conclusion}

Our study suggests that lung imaging performed during routine EFAST evaluations in trauma patients is not an accurate diagnostic tool for patients with active COVID-19 infection. This is true when EFAST imaging is evaluated by both trainees and attending radiologists. At a large academic center, specifically a high-volume level 1 trauma center, the use of EFAST is not a reliable screening tool for COVID19 infections, and standard diagnostic imagining and PCR tests remain more reliable diagnostic tools in these settings. Trauma activations are high acuity situations that require a significant number of healthcare workers. Exposure to active infection in a patient with an unknown COVID-19 status is of concern. However, due to concomitant injuries and the technique of EFAST imaging, this tool cannot be expanded to exclude active infection in trauma patients undergoing assessment. Previous findings of the diagnostic accuracy of US for detection of the virus should not be expanded to the trauma population.

Acknowledgements The authors wish to thank Stephanie Vega from University of Colorado Hospital at the Anschutz Medical Center and Karin Winery at Children's Hospital Colorado for their assistance with providing data from their respective trauma registries.

\section{Declarations}

Ethics approval The Colorado Multiple Institutional Review Board (COMIRB) approved this study. As EFAST is a standard part of trauma evaluation, waiver of consent was approved by the COMIRB.

Conflict of interest The authors declare that they have no conflict of interest.

\section{References}

1. Kim H, Hong H, Yoon SH (2020) Diagnostic performance of CT and reverse transcriptase polymerase chain reaction for coronavirus disease 2019: a meta-analysis. Radiology 296(3):E145-E155. https://doi.org/10.1148/radiol.2020201343

2. Kanne JP, Little BP, Chung JH, Elicker BM, Ketai LH (2020) Essentials for radiologists on COVID-19: an update- radiology scientific expert panel. Radiology 296(2):E113-E114. https://doi. org/10.1148/radiol.2020200527

3. Sofia S, Boccatonda A, Montanari M, Spampinato M, D'ardes D, Cocco G, Accogli E, Cipollone F, Schiavone C (2020) Thoracic ultrasound and SARS-COVID: a pictorial essay. J Ultrasound 23(2):217-221

4. Smith MJ, Hayward SA, Innes SM, Miller ASC (2020) Pointof-care lung ultrasound in patients with COVID-19 - a narrative review. Anaesthesia 75(8):1096-1104. https://doi.org/10.1111/ anae. 15082

5. Rizzetto F, Perillo N, Artioli D, Travaglini F, Cuccia A, Zannoni S, Tombini V, Di Domenico SL, Albertini V, Bergamaschi M, Cazzaniga M, De Mattia C, Torresin A, Vanzulli A, Niguarda COVID-19 Working Group (2021) Correlation between lung ultrasound and chest CT patterns with estimation of pulmonary burden in COVID-19 patients. Eur J Radiol 138:109650. https:// doi.org/10.1016/j.ejrad.2021.109650

6. Lieveld AWE, Kok B, Schuit FH, Azijli K, Heijmans J, van Laarhoven A, Assman NL, Kootte RS, Olgers TJ, Nanayakkara PWB, Bosch FH (2020) Diagnosing COVID-19 pneumonia in a pandemic setting: lung ultrasound versus CT (LUVCT) - a multicentre, prospective, observational study. ERJ Open Res 
21;6(4):00539-2020. https://doi.org/10.1183/23120541.005392020. eCollection 2020 Oct.

7. Colombi D, Petrini M, Maffi G, Villani GD, Bodini FC, Morelli N, Milanese G, Silva M, Sverzellati N, Michieletti E (2020) Comparison of admission chest computed tomography and lung ultrasound performance for diagnosis of COVID-19 pneumonia in populations with different disease prevalence. Eur J Radiol 133:109344. https://doi.org/10.1016/j.ejrad.2020.109344

8. Colorado State Emergency Operations Center (2021) Colorado Department of Public Health \& Environment. Colorado COVID19 Data, https://covid19.colorado.gov/data. Accessed 21 July 2021.

9. Ianniello S, Di Giacomo V, Sessa B, Miele V (2014) First-line sonographic diagnosis of pneumothorax in major trauma: accuracy of e-FAST and comparison with multidetector computed tomography. Radiol Med 119(9):674-680. https://doi.org/10. 1007/s11547-014-0384-1

10. Buonsenso D, Piano A, Raffaelli F, Bonadia N, de Gaetano Donati K, Franceschi F (2020) Point-of-care lung ultrasound findings in novel coronavirus disease-19 pnemoniae: a case report and potential applications during COVID-19 outbreak. Eur Rev Med Pharmacol Sci 24(5):2776-2780. https://doi.org/10.26355/eurrev_ 202003_20549

11. Haak SL, Renken IJ, Cara Jager L, Lameijer H, van der Kolk BBY (2020) Diagnostic accuracy of point-of-care lung ultrasound in COVID-19. Emerg Med J 18:emermed-2020-210125. https://doi. org/10.1136/emermed-2020-210125. Online ahead of print.

12. Nuñez JH, Sallent A, Lakhani K, Guerra-Farfan E, Vidal N, Ekhtiari S, Minguell J (2020) Impact of the COVID-19 pandemic on an emergency traumatology service: experience at a tertiary trauma centre in Spain. Injury 51(7):1414-1418. https://doi.org/ 10.1016/j.injury.2020.05.016

13. Shi Y, Kvasnovsky C, Khan S, Jain S, Sargeant D, Lamoshi A, Prince J, Sathya C (2021) Impact of the COVID-19 pandemic on trauma activations at a pediatric level 1 trauma center in New York. Pediatr Surg Int 1-6. https://doi.org/10.1007/s00383-02104962-7. Online ahead of print.

14. Sutherland M, McKenny M, Elkbuli A (2020) Vehicle related injury patterns during the COVID-19 pandemic: what has changed? Am J Emerg Med 38(9):1710-1714. https://doi.org/10. 1016/j.ajem.2020.06.006

15. Giudici R, Lancioni A, Gay H, Bassi G, Chiara O, Mare C, Latronico N, Pesenti A, Faccincani R, Cabrini L, Fumagalli R, Chieregato A, Briani L, Sammartano F, Sechi G, Zoli A, Pagliosa A, Foti G, Borotto E, Palo A, Valoti O, Botteri M, Carlucci M, Reitano E, Bini R (2021) Impact of the COVID-19 outbreak on severe trauma trends and healthcare system reassessment in Lombardia, Italy: an analysis from the regional trauma registry. World J Emerg Surg 16(1):39. https://doi.org/10.1186/s13017-021-00383-y

16. Staub LJ, Biscaro RRM, Kaszubowski E, Maurici R (2018) Chest ultrasonography for the emergency diagnosis of traumatic pneumothorax and haemothorax: a systematic review and meta-analysis. Injury 49(3):457-466. https://doi.org/10.1016/j.injury.2018. 01.033
17. Rouhezamin MR, Paydar S, Haseli S (2020) COVID-19 or pulmonary contusion? A diagnostic dilemma Acad Radiol 27(6):894895. https://doi.org/10.1016/j.acra.2020.04.008

18. Chen LR, Chen ZX, Liu YC, Peng L, Zhang Y, Xu Q, Lin Q, Tao YM, Wu H, Yin S, Hu YJ (2020) Pulmonary contusion mimicking COVID-19: a case report. World J Clin Cases 8(8):1554-1560. https://doi.org/10.12998/wjcc.v8.i8.1554

19. Wang Y, Zeng C, Dong L, Liu C, Cai Y, Zhang N, Fu X (2020) Pulmonary contusion during the COVID-19 pandemic: challenges in diagnosis and treatment. Surg Today 50(9):1113-1116. https:// doi.org/10.1007/s00595-020-02081-9

20. Abbasi S, Shaker H, Zareiee F, Farsi D, Hafezimoghadam P, Rezai M, Mahshidfar B, Mofidi M (2018) Screening performance of ultrasonographic B-lines in detection of lung contusion following blunt trauma; a diagnostic accuracy study. Emerg (Tehran) 6(1):e55

21. Soldati G, Smargiassi A, Inchingolo R, Buonsenso D, Perrone T, Briganti DF, Perlini S, Torri E, Mariani A, Mossolani EE, Tursi F, Mento F, Demi L (2020) Is there a role of lung ultrasound during the COVID-19 pandemic? J Ultrasound Med 39(7):1459-1462. https://doi.org/10.1002/jum.15284

22. Chung M, Bernheim A, Mei X, Zhang N, Huang M, Zeng X, Cui J, Xu W, Yang Y, Fayad ZA, Jacobi A, Li K, Li S, Shan H (2020) CT imaging features of 2019 novel coronavirus (2019-nCoV). Radiology 295(1):202-207. https://doi.org/10.1148/radiol.20202 00230

23. Zanobetti M, Coppa A, Nazerian P, Grifoni S, Scorpiniti M, Innocenti F, Conti A, Bigiarini S, Gualtieri S, Casula C, Ticali PF, Pini R (2018) Chest abdominal-focused assessment sonography for trauma during the primary survey in the emergency department: the CA-FAST protocol. Eur J Trauma Emerg Surg 44(6):805-810. https://doi.org/10.1007/s00068-015-0620-y

24. Nandipati KC, Allamaneni S, Kakarla R, Wong A, Richards N, Satterfield J, Turner JW, Sung KJ (2011) Extended focused assessment with sonography for trauma (EFAST) in the diagnosis of pneumothorax: experience at a community based level I trauma center. Injury 42(5):511-514. https://doi.org/10.1016/j.injury. 2010.01.105

25. Song F, Shi N, Shan F, Zhang Z, Shen J, Lu H, Ling Y, Jiang Y, Shi Y (2020) Emerging 2019 novel coronavirus (2019-nCoV) pneumonia. Radiology 295(1):210-217. https://doi.org/10.1148/ radiol.2020200274

26. Pan F, Ye T, Sun P, Gui S, Liang B, Li L, Zheng D, Wang J, Hesketh RL, Yang L, Zheng C (2020) Time course of lung changes at chest CT during recovery from coronavirus disease 2019 (COVID-19). Radiology 295(3):715-721. https://doi.org/10. 1148/radiol.2020200370

Publisher's note Springer Nature remains neutral with regard to jurisdictional claims in published maps and institutional affiliations. 


\section{Authors and Affiliations}

\section{Jose Diaz-Miron ${ }^{1}$ - Marina L. Reppucci ${ }^{1}$. Jason Weinman ${ }^{2} \cdot$ Alexander Kaizer $^{3}$ - Aparna Annam ${ }^{2}$. Jonathan Orsborn $^{4} \cdot$ Lauren Steward $^{5} \cdot$ Juliana Wilson $^{6} \cdot$ Denis Bensard $^{1}$}

1 Division of Pediatric Surgery, Department of Surgery, Children's Hospital Colorado, University of Colorado School of Medicine, 13123 E 16th Ave, B-323, Aurora, CO 80045, USA

2 Division of Pediatric Radiology, Department of Radiology, University of Colorado School of Medicine, Aurora, CO, USA

3 Center for Innovative Design and Analysis, Department of Biostatistics and Informatics, Colorado School of Public Health, University of Colorado, Aurora, CO, USA
4 Division of Pediatric Emergency Medicine, Department of Emergency Medicine, University of Colorado School of Medicine, Aurora, CO, USA

5 Division of Gastrointestinal, Trauma, and Endocrine Surgery, Department of Surgery, University of Colorado School of Medicine, Aurora, CO, USA

6 Department of Emergency Medicine, University of Colorado School of Medicine, Aurora, CO, USA 